\title{
Existence Results for Vector Mixed Quasi-Complementarity Problems
}

\author{
Suhel Ahmad Khan ${ }^{1}$ and Naeem Ahmad ${ }^{2}$ \\ ${ }^{1}$ Department of Mathematics, BITS-Pilani, Dubai Campus, P.O. Box 345055, Dubai, UAE \\ ${ }^{2}$ Department of Mathematics, Al-Jouf University, P.O. Box 2014, Skaka, Saudi Arabia
}

Correspondence should be addressed to Suhel Ahmad Khan; khan.math@gmail.com

Received 27 September 2012; Revised 16 January 2013; Accepted 17 January 2013

Academic Editor: Kaleem R. Kazmi

Copyright (C) 2013 S. A. Khan and N. Ahmad. This is an open access article distributed under the Creative Commons Attribution License, which permits unrestricted use, distribution, and reproduction in any medium, provided the original work is properly cited.

We introduce strong vector mixed quasi-complementarity problems and the corresponding strong vector mixed quasi-variational inequality problems. We establish equivalence between strong mixed quasi-complementarity problems and strong mixed quasivariational inequality problem in Banach spaces. Further, using KKM-Fan lemma, we prove the existence of solutions of these problems, under pseudomonotonicity assumption. The results presented in this paper are extensions and improvements of some earlier and recent results in the literature.

\section{Introduction}

In 1980, Giannessi [1] introduced vector variational inequalities in a finite-dimensional Euclidean space. Motivated by Giannessi [1], Chen and Cheng [2] studied vector variational inequalities in infinite-dimensional Euclidean space and applied them to the vector optimization problems. Since then, vector variational inequalities and their generalizations have been studied and applied to vector optimization problems, vector complementarity problems, game theory, and so forth; see, for example, [1-20] and references therein. It is well known that the complementarity problems are closely related to variational inequality problems. Complementarity theory is introduced by Lemke [21] and Cottle and Dantzig [5]. It has emerged as an active and interesting field for researcher with wide range of applications in pure and applied sciences. Complementarity problems have been extended and generalized in various directions to study a large class of problems arising in industry, finance, optimization, physical, mathematical and engineering sciences; see, for example [4-12, 14, 15, 20$]$. Recently, vector complementarity problems and their relations with vector variational inequality problems have been investigated under pseudomonotone-type conditions and positiveness-type conditions; see, for example [6, 8-10, 20]. However, to the best of our knowledge, only a few existence results on the strong version of the vector variational inequality and vector complementarity problems were established.

Recently, Huang et al. [12] discussed equivalence results among a vector complementarity problem, a vector variational inequality problem, a vector optimization problem, and weak minimal element problem, under some monotonicity conditions and some inclusive-type conditions in ordered Banach spaces. In 2005, Huang and Fang [9] introduced several classes of strong vector F-complementarity problems and give some existence results for these problems in Banach spaces and discussed the least element problems of feasible sets and presented their relations with the strong vector Fcomplementarity problems.

Very recently, Khan [22] introduced and studied a generalized vector implicit Quasi-Complementarity problem and generalized vector implicit quasi variational inequality problem. He investigated the nonemptiness and closedness of solution sets of these problems and proved that solution sets of both the problems are equivalent to each other under some suitable conditions.

Inspired and motivated by the work going in this direction, in this paper we introduce and analyze a new class of strong vector Quasi-Complementarity problem and the corresponding strong vector mixed quasi variational inequality problem in the setting of Banach space and establish 
equivalence results between them. By using the KKM-Fan lemma, we derive the existence of solutions of strong vector mixed quasi variational inequalities under pseudomonotonicity assumption and show that the solution of the strong vector mixed quasi variational inequality is equivalent to the solution of strong vector mixed Quasi-Complementarity problems under suitable conditions. The results presented in this paper are the generalization and improvement of existing works of $[6,7,9,11,15]$.

\section{Preliminaries}

Throughout this paper unless otherwise stated let $X$ and $Y$ be two real Banach spaces. Let $K$ be a nonempty, closed, convex subset of a real Banach space $X$. A nonempty subset $P \subset Y$ is called convex, pointed, connected, and reproduced cone, respectively, if it satisfies the following conditions: (i) $\lambda P \subseteq P$, for all $\lambda>0$ and $P+P \subseteq P$; (ii) $P \cap-P=\{0\}$; (iii) $P \cup-P=X$; (iv) $P-P=X$.

Given $P$ in $Y$, we can define the relations " $\leq_{P}$ " and " $\not_{P}$ " as follows:

$$
\begin{gathered}
x \leq_{P} y \Longleftrightarrow y-x \in P, \\
x \Varangle_{P} y \Longleftrightarrow y-x \notin P, \quad \forall x, y \in Y .
\end{gathered}
$$

If " $\leq_{P}$ " is a partial order, then $\left(Y, \leq_{P}\right)$ is called a Banach space ordered by $P$. Let $L(X, Y)$ denote the space of all continuous linear mappings from $X$ into $Y$.

Now, we recall the following concepts and results needed in this paper.

Definition 1. A mapping $f: K \times K \rightarrow Y$ is said to be $P$-convex in first argument, if

$$
\begin{aligned}
f(t x+(1-t) y, z) \leq_{P} t f(x, z)+(1-t) f(y, z), \\
\forall x, y, z \in K, \quad t \in[0,1] .
\end{aligned}
$$

Definition 2. Let $T: K \rightarrow L(X, Y)$ and $F: K \times K \rightarrow Y$ be the two nonlinear mappings. $T$ is said to be monotone with respect to $F$ if

$$
\begin{array}{r}
\langle T x-T y, x-y\rangle+F(y, x)-F(x, x) \geq_{P} 0, \\
\forall x, y \in K .
\end{array}
$$

Definition 3. Let $T: K \rightarrow L(X, Y)$ and $F: K \times K \rightarrow Y$ be the two nonlinear mappings. $T$ is said to be pseudomonotone with respect to $F$ if, for any given $x, y \in K$,

$$
\begin{aligned}
\langle T x, y-x\rangle+F(y, x)-F(x, x) & \Varangle_{P \backslash\{0\}} 0 \\
& \Longrightarrow\langle T y, y-x\rangle+F(y, x)-F(x, x) \geq_{P} 0 .
\end{aligned}
$$

Remark 4. Every monotone with respect to $F$ is pseudomonotone with respect to $F$ but converse does not hold in general. Definition 3 is vector version of $\theta$-pseudomonotonicity studied by Kazmi et al. in $[23,24]$.
Example 5. Let $X=\mathbb{R}, K=\mathbb{R}_{+}, Y=\mathbb{R}^{2}, P=\mathbb{R}_{+}^{2}$, and

$$
\begin{gathered}
T(x)=\left(\begin{array}{c}
0 \\
\sin x \cos x
\end{array}\right), \\
F(y, x)=\left(\begin{array}{c}
y+x \\
y+x
\end{array}\right), \quad \forall x, y \in K .
\end{gathered}
$$

Now,

$$
\begin{aligned}
\langle T x, y-x\rangle+F(y, x)-F(x, x) & \\
= & \left(\begin{array}{c}
y-x \\
(1+\sin x \cos x)(y-x)
\end{array}\right) \$_{P \backslash\{0\}} 0 .
\end{aligned}
$$

We have $y \geq x$. It follows that

$$
\begin{aligned}
\langle T y, y-x\rangle+F(y, x)-F(x, x) \\
=\left(\begin{array}{c}
y-x \\
(1+\sin y \cos y)(y-x)
\end{array}\right) \geq_{P} 0 .
\end{aligned}
$$

So, $T$ is pseudomonotone with respect to $F$. However, for $x=$ $\pi$ and $y=\pi / 2$, it follows that

$$
\langle T x-T y, x-y\rangle+F(y, x)-F(x, x)=\left(\begin{array}{r}
-\frac{\pi}{2} \\
-\frac{\pi}{2}
\end{array}\right) \nsupseteq_{P} 0 .
$$

This shows that $T$ is not a monotone with respect to $F$.

Definition 6. A mapping $T: K \rightarrow L(X, Y)$ is said to be hemicontinuous if, for any $x, y \in K$, the mapping $t \mapsto\langle T(x+$ $t(y-x)), y-x>$ is continuous at $0^{+}$.

Definition 7. A mapping $F: K \times K \rightarrow Y$ is said to be positively homogeneous in first argument, if $F(t x, y)=t F(x, y)$ for all $x, y \in K$ and $t \geq 0$.

Definition 8 . Let $K$ be a nonempty subset of a topological vector space $X$. A set-valued map $T: K \rightarrow 2^{X}$ is said to be a KKM mapping if, for each nonempty finite subset $\left\{x_{1}, \ldots, x_{n}\right\} \subset K, \operatorname{co}\left\{x_{1}, \ldots, x_{n}\right\} \subset \bigcup_{i=1}^{n} T\left(x_{i}\right)$, where co denotes the convex hull.

Lemma 9 (KKM-Fan Lemma (see [25])). Let $K$ be a nonempty subset of Hausdorff topological vector space $X$. Let $T: K \rightarrow 2^{X}$ be a KKM-mapping such that for each $x \in$ $K, T(x)$ is closed and for at least one $x \in K, T(x)$ is compact, then

$$
\bigcap_{x \in K} T(x) \neq \emptyset
$$

\section{Strong Vector Mixed Quasi-Complementarity Problems}

Throughout this section, let $X$ be a real Banach space and let $K \subseteq X$ be a nonempty, closed, and convex subset of $X$. Let $\left(Y, \leq_{P}\right)$ be an ordered Banach space induced by a pointed, closed, convex cone $P$ with nonempty interior. Let 
$T: K \rightarrow L(X, Y)$ and $F: K \times K \rightarrow Y$ be the two nonlinear mappings. In this paper, we consider the following strong vector mixed Quasi-Complementarity problems:

(i) Strong vector mixed Quasi-Complementarity problem (SVMQCP) $)_{1}$ :

Find $x \in K$ such that $\langle T x, x\rangle+F(x, x) \Varangle_{P \backslash\{0\}} 0$, $\langle T x, y\rangle+F(y, x) \Varangle_{P \backslash\{0\}} 0$, for all $y \in K$.

(ii) Strong vector mixed Quasi-Complementarity problem (SVMQCP) $)_{2}$ :

Find $x \in K$ such that $\langle T x, x\rangle+F(x, x)=0$, $\langle T x, y\rangle+F(y, x) \Varangle_{P \backslash\{0\}} 0$, for all $y \in K$.

Closely related to (SVMQCP) $)_{1}$ and (SVMQCP) $)_{2}$ problems, we consider the following strong vector mixed quasi variational inequality problem:

Strong vector mixed quasi variational inequality problem (SVMQVIP):

Find $x \in K$ such that $\langle T x, y-x\rangle+F(y, x)-$ $F(x, x) \Varangle_{P \backslash\{0\}} 0$, for all $y \in K$.

The strong vector mixed quasi variational inequality problem (SVMQVIP) is the generalization and extension of many previously known vectors as well as scalar mixed quasi variational inequalities. For the formulation, numerical results, existence results, sensitivity analysis, and dynamical aspects of the mixed quasi variational inequalities, see $[3,5$, $7,16,17]$ and the references therein.

Remark 10. (1) If $Y=\mathbb{R}$ and $P=\mathbb{R}^{+}$, then (SVMQCP) $)_{1}$ and $(\mathrm{SVMQCP})_{2}$ and (SVMQVIP) reduce, respectively, to the mixed Quasi-Complementarity problem (MQCP):

(MQCP) Find $x \in K$ such that $\langle T x, y-x\rangle+F(x, x)=$ $0,\langle T x, y\rangle+F(y, x) \geq 0$, for all $y \in K$

and mixed quasi variational inequality problem (MQVIP):

(MQVIP) Find $x \in K$ such that $\langle T x, y-x\rangle+F(y, x)-$ $F(x, x) \geq 0$, for all $y \in K$,

which were introduced and studied by Farazjadeh et al. [7].

(2) If $F=0$, then $(\mathrm{SVMQCP})_{1}$ and (SVMQCP $)_{2}$ reduce to the following strong vector complementarity problems (SVCP):

$(\mathrm{SVCP})_{1}$ Find $x \in K$ such that $\langle T x, x\rangle \nsupseteq_{P \backslash\{0\}} 0$, $\langle T x, y\rangle \Varangle_{P \backslash\{0\}} 0$, for all $y \in K$,

$(\mathrm{SVCP})_{2}$ Find $x \in K$ such that $\langle T x, x\rangle=0$, $\langle T x, y\rangle \Varangle_{P \backslash\{0\}} 0$, for all $y \in K$

and (SVMQVIP) reduces to the following strong vector variational inequality problem (SVVIP):

(SVVIP) Find $x \in K$ such that $\langle T x, y-x\rangle \Varangle_{P \backslash\{0\}} 0$, for all $y \in K$.
First, we will investigate the equivalences among $(\mathrm{SVMQCP})_{1}$ and (SVMQCP) $)_{2}$ and (SVMQVIP), under some suitable assumptions.

Theorem 11. (i) Suppose that $\langle T z, z\rangle+F(z, z) \in P \cup(-P)$, for all $z \in K$. If $x$ solves (SVMQCP $)_{1}$ then $x$ solves (SVMQVIP).

(ii) Let $F: K \times K \rightarrow Y$ satisfy $F(2 x, y)=2 F(x, y)$, for all $x, y \in K$ and $\langle T z, z\rangle+F(z, z) \in P \cup(-P)$, for all $z \in K$. If $x$ solves (SVMQVIP) then $x$ also solves $(S V M Q C P)_{1}$.

Proof. (i) Let $x \in K$ be the solution of (SVMQCP) $)_{1}$. Then $x \in K$ such that

$$
\begin{gathered}
\langle T x, x\rangle+F(x, x) \nsupseteq_{P \backslash\{0\}} 0, \\
\langle T x, y\rangle+F(y, x) \Varangle_{P \backslash\{0\}} 0, \quad \forall y \in K .
\end{gathered}
$$

Substituting $y=x$ in Inclusion (11), we get

$$
\langle T x, x\rangle+F(x, x) \Varangle_{P \backslash\{0\}} 0 .
$$

Since $\langle T z, z\rangle+F(z, z) \in P \cup(-P)$, for all $z \in K$, we have

$$
\langle T x, x\rangle+F(x, x) \geq_{P} 0 \quad \text { or } \quad\langle T x, x\rangle+F(x, x) \leq_{P} 0 .
$$

From Inclusions (10), (12), and (13), we have

$$
\langle T x, x\rangle+F(x, x)=0 .
$$

From (11) and (14), we have

$$
\begin{aligned}
& \langle T x, y-x\rangle+F(y, x)-F(x, x) \\
& =\langle T x, y\rangle+F(y, x)-\langle T x, x\rangle-F(x, x) \\
& =\langle T x, y\rangle+F(y, x) \\
& \quad \Varangle_{P \backslash\{0\}} 0,
\end{aligned}
$$

for all $y \in K$. Thus, $x \in K$ is the solution of (SVMQVIP).

(ii) Now, let $x \in K$ be the solution of (SVMQVIP), then

$$
\langle T x, y-x\rangle+F(y, x)-F(x, x) \varliminf_{P \backslash\{0\}} 0, \quad \forall y \in K .
$$

Since $F(2 x, y)=2 F(x, y)$, for all $x, y \in K$, therefore it follows that $F(0, y)=0$, for all $y \in K$. By substituting $y=2 x$ and $y=0$, respectively, in (16), we get

$$
\begin{aligned}
& \langle T x, x\rangle+F(x, x) ⿱_{P \backslash\{0\}} 0, \\
& \langle T x, x\rangle+F(x, x) \pm_{P \backslash\{0\}} 0 .
\end{aligned}
$$

Since $\langle T z, z\rangle+F(z, z) \in P \cup(-P)$, for all $z \in K$, we have

$$
\langle T x, x\rangle+F(x, x) \geq_{P} 0 \quad \text { or } \quad\langle T x, x\rangle+F(x, x) \leq_{P} 0 .
$$

From (17) and (18), we have

$$
\langle T x, x\rangle+F(x, x)=0 .
$$

By using Inclusions (16) and (19), we have

$$
\begin{aligned}
&\langle T x, y\rangle+F(y, x) \\
&=\langle T x, y-x\rangle+F(y, x)-F(x, x) \\
&+\langle T x, x\rangle+F(x, x) \\
&=\langle T x, y-x\rangle+F(y, x)-F(x, x) \\
& \Varangle_{P \backslash\{0\}} 0,
\end{aligned}
$$


for all $y \in K$, which implies that $x$ solves (SVMQCP) $)_{1}$.

Remark 12. The condition $F(2 x, y)=2 F(x, y)$, for all $x, y \in$ $K$ holds if $F$ is positively homogeneous; that is, $F(t x, y)=$ $t F(x, y)$ for all $t \geq 0$. Hence, Theorem 11 generalizes and improves the theorems in $[6,9,11,14,15]$.

Here we give an example of a function $F$, which satisfies the condition $F(2 x, y)=2 F(x, y)$, for all $x, y \in K$ but not a positively homogeneous, which implies that previously known results in $[6,9,11,14,15]$ cannot be applied.

Example 13. Let $F: \mathbb{R} \times \mathbb{R} \rightarrow \mathbb{R}$, defined by

$$
F(x, y)= \begin{cases}2 x, & \text { if } x \text { rational, } \\ 0, & \text { if } x \text { irrational. }\end{cases}
$$

Then $F$ satisfies $F(2 x, y)=2 F(x, y)$ but it is not positively homogeneous.

Theorem 14. (a) If $x$ solves Problem $(S V M Q C P)_{2}$ then $x$ solves (SVMQVIP).

(b) Let $F: K \times K \rightarrow Y$ satisfy $F(2 x, y)=2 F(x, y)$, for all $x, y \in K$ and $\langle T z, z\rangle+F(z, z) \in P \cup(-P)$, for all $z \in K$. If $x$ solves (SVMQVIP) then $x$ solves $(S V M Q C P)_{2}$.

Proof. (a) Let $x \in K$ be the solution of (SVMQCP) $)_{2}$. Then $x \in K$ such that

$$
\begin{gathered}
\langle T x, x\rangle+F(x, x)=0, \\
\langle T x, y\rangle+F(y, x) \not_{P \backslash\{0\}} 0, \quad \forall y \in K .
\end{gathered}
$$

Now,

$$
\begin{aligned}
& \langle T x, y-x\rangle+F(y, x)-F(x, x) \\
& =\langle T x, y\rangle+F(y, x)-\langle T x, x\rangle-F(x, x) \\
& =\langle T x, y\rangle+F(y, x) \\
& \quad \$_{P \backslash\{0\}} 0,
\end{aligned}
$$

for all $y \in K$. Thus, $x \in K$ is the solution of (SVMQVIP).

(b) Now, let $x \in K$ be the solution of (SVMQVIP), then

$$
\langle T x, y-x\rangle+F(y, x)-F(x, x) \Varangle_{P \backslash\{0\}} 0, \quad \forall y \in K .
$$

Since $F(2 x, y)=2 F(x, y)$, for all $x, y \in K$, therefore it follows that $F(0, y)=0$, for all $y \in K$. By substituting $y=2 x$ and $y=0$, respectively, in (24), we get

$$
\begin{aligned}
& \langle T x, x\rangle+F(x, x) ⿱_{P \backslash\{0\}} 0, \\
& \langle T x, x\rangle+F(x, x) \unlhd_{P \backslash\{0\}} 0 .
\end{aligned}
$$

Since $\langle T z, z\rangle+F(z, z) \in P \cup(-P)$, for all $z \in K$, we have

$$
\langle T x, x\rangle+F(x, x) \geq_{P} 0 \quad \text { or } \quad\langle T x, x\rangle+F(x, x) \leq_{P} 0 .
$$

From (25) and (26), we have

$$
\langle T x, x\rangle+F(x, x)=0 .
$$

By using (27), we have

$$
\begin{aligned}
&\langle T xy\rangle+F(y, x) \\
&=\langle T x, y-x\rangle+F(y, x)-F(x, x) \\
&+\langle T x, x\rangle+F(x, x) \\
&=\langle T x, y-x\rangle+F(y, x)-F(x, x) \\
& \$_{P \backslash\{0\}} 0,
\end{aligned}
$$

for all $y \in K$. Then (27) and (28) imply that $x$ solves $(\mathrm{SVMQCP})_{2}$.

\section{Existence Results}

First, we prove following Minty-type lemma with the help of pseudomonotone mapping with respect to $F$.

Lemma 15. Let $F: K \times K \rightarrow Y$ be $P$-convex in first argument and let $T: K \rightarrow L(X, Y)$ be a hemicontinuous mapping and pseudomonotone with respect to $F$. Then the following two problems are equivalent:

(A)

$x \in K,\langle T x, y-x\rangle+F(y, x)-F(x, x) \Varangle_{P \backslash\{0\}}, \quad \forall y \in K$,

$x \in K,\langle T y, y-x\rangle+F(y, x)-F(x, x) \geq_{P} 0, \quad \forall y \in K$.

Proof. (29) $\Rightarrow$ (30). The result directly follows from pseudomonotonicity with respect to $F$.

Now, (30) $\Rightarrow(29)$. For any given $y \in K$, we know that $y_{t}=t y+(1-t) x \in K$, for all $t \in(0,1)$, as $K$ is convex. Since $x \in K$ is a solution of problem (30), so for each $x \in K$, it follows that

$$
\left\langle T y_{t}, y_{t}-x\right\rangle+F\left(y_{t}, x\right)-F(x, x) \geq_{P} 0 .
$$

Now, we have

$$
\begin{aligned}
& t\left\langle T y_{t}, y-x\right\rangle+t(F(y, x)-F(x, x)) \\
& \quad \geq\left\langle T y_{t}, y_{t}-x\right\rangle+F\left(y_{t}, x\right)-F(x, x) \\
& \quad \geq_{P} 0 .
\end{aligned}
$$

For $t>0$, we get

$$
\left\langle T y_{t}, y-x\right\rangle+F(y, x)-F(x, x) \geq_{P} 0 .
$$

Since $T$ is hemicontinuous and $P$ is closed, letting $t \rightarrow 0^{+}$in inclusion (33), we get

$$
\langle T x, y-x\rangle+F(y, x)-F(x, x) \geq_{P} 0, \quad \forall y \in K .
$$

Hence,

$$
\langle T x, y-x\rangle+F(y, x)-F(x, x) \$_{P \backslash\{0\}}, \quad \forall y \in K .
$$

Therefore, $x \in K$ is solution of problem (29). This completes the proof. 
Now, with the help of Lemma 15, we have following existence theorem for (SVMQVIP).

Theorem 16. Let $X$ be real reflexive Banach space and let $Y$ be a Banach space. Let $K \subset X$ be a nonempty, bounded, closed, and convex subset of $X$. Let $F: K \times K \rightarrow Y$ be $P$-convex and upper semicontinuous in first and second arguments, respectively. Let $T: K \rightarrow L(X, Y)$ be hemicontinuous and pseudomonotone with respect to F. Then (SVMQVIP) has solution.

Proof. Define two set-valued mappings $F, G: K \rightarrow 2^{K}$ as follows:

$$
\begin{aligned}
& G(y)=\{x \in K:\langle T x, y-x\rangle
\end{aligned}
$$

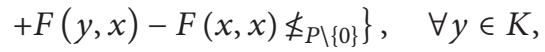

$$
\begin{aligned}
& H(y)=\{x \in K:\langle T y, y-x\rangle \\
& \left.+F(y, x)-F(x, x) \geq_{P} 0\right\}, \quad \forall y \in K .
\end{aligned}
$$

$G(y)$ and $H(y)$ are nonempty, since $y \in G(y) \cap H(y)$. We claim that $G$ is a KKM mapping. If this is not true, then there exists a finite set $\left\{y_{1}, \ldots, y_{n}\right\} \subset K$ and $t_{i} \geq 0, i=1, \ldots, n$ with $\sum_{i=1}^{n} t_{i}=1$ such that $y=\sum_{i=1}^{n} t_{i} y_{i} \notin \bigcup_{i=1}^{n} G\left(y_{i}\right)$. Now, by the definition of $G$, we have

$$
\left\langle T y, y_{i}-y\right\rangle+F\left(y_{i}, y\right)-F(y, y) \leq_{P \backslash\{0\}}, \quad i=1, \ldots, n .
$$

Now, we have

$$
\begin{aligned}
0 & =\langle T y, y-y\rangle+F(y, y)-F(y, y) \\
& =\left\langle T y, \sum_{i=1}^{n} t_{i} y_{i}-y\right\rangle+F\left(\sum_{i=1}^{n} t_{i} y_{i}, y\right)-F(y, y) \\
& =\sum_{i=1}^{n} t_{i}\left[\left\langle T y, y_{i}-y\right\rangle+F\left(y_{i}, y\right)-F(y, y)\right] \\
& \leq_{P \backslash\{0\}},
\end{aligned}
$$

which is not possible. Thus, our claim is verified. So $G$ is a KKM mapping.

Now, since $T$ is pseudomonotone with respect to $F$, therefore $G(y) \subset H(y)$ for every $y \in K$ and so $H$ is also a KKM mapping. Now we claim that for each $y \in K, H(y) \subset K$ is closed in the weak topology of $X$.

Indeed, suppose $\bar{x} \in \overline{H(y)}^{w}$, the weak closure of $H(y)$. Since $X$ is reflexive, there is a sequence $\left\{x_{n}\right\}$ in $H(y)$ such that $\left\{x_{n}\right\}$ converges weakly to $\bar{x} \in K$. Then

$$
\left\langle T y, y-x_{n}\right\rangle+F\left(y, x_{n}\right)-F\left(x, x_{n}\right) \geq_{P} 0 .
$$

Since $F(y, \cdot)$ is upper semicontinuous and $P$ is closed, therefore,

$$
\langle T y, y-\bar{x}\rangle+F(y, \bar{x})-F(x, \bar{x}) \geq_{P} 0
$$

and so $\bar{x} \in H(y)$. This shows that $H(y)$ is weakly closed, for each $y \in K$. Our claim is then verified. Since $X$ is reflexive and $K \subset X$ is nonempty, bounded, closed and convex, $K$ is a weakly compact subset of $X$ and so $H(y)$ is also weakly compact. According to Lemma 9 (KKM-Fan Lemma),

$$
\bigcap_{y \in K} H(y) \neq \emptyset .
$$

This implies that there exists $x \in K$ such that

$$
\langle T y, y-x\rangle+f(y, x)-F(x, x) \geq_{P} 0, \quad \forall y \in K .
$$

Therefore by Lemma 15, we conclude that there exists $x \in K$ such that

$$
\langle T x, y-x\rangle+F(y, x)-F(x, x) \Varangle_{P \backslash\{0\}}, \quad \forall y \in K .
$$

This completes the proof.

Theorem 17. Let $F: K \times K \rightarrow Y$ satisfy $F(2 x, y)=2 F(x, y)$, for all $x, y \in K$. If all the assumptions of Theorem 16 hold, then $(S V M Q C P)_{1}$ is solvable. In addition, if $\langle T z, z\rangle+F(z, z) \in P \cup$ $(-P)$, for all $z \in K$, then $(S V M Q C P)_{2}$ is solvable.

Proof. The conclusion follows directly from Theorems 11, 14, and 16.

\section{References}

[1] F. Giannessi, "Theorems of alternative, quadratic programs and complementarity problems," in Variational Inequalities and Complementarity Problems, R. W. Cottle, F. Gianneesi, and J. L. Lions, Eds., pp. 151-186, Wiley, Chichester, UK, 1980.

[2] G. Y. Chen and G. M. Cheng, "Vector variational inequalities and vector optimization," in Lecture Notes in Economics and Mathematical Systems, vol. 285, pp. 408-416, Springer, Berlin, Germany, 1987.

[3] A. Bnouhachem and M. A. Noor, "Numerical method for general mixed quasi-variational inequalities," Applied Mathematics and Computation, vol. 204, no. 1, pp. 27-36, 2008.

[4] G. Y. Chen and X. Q. Yang, “The vector complementary problem and its equivalences with the weak minimal element in ordered spaces," Journal of Mathematical Analysis and Applications, vol. 153, no. 1, pp. 136-158, 1990.

[5] R. W. Cottle and G. B. Dantzig, "Complementary pivot theory of mathematical programming," Linear Algebra and its Applications, vol. 1, pp. 163-185, 1968.

[6] Y.-P. Fang and N.-J. Huang, "The vector F-complementary problems with demipseudomonotone mappings in Banach spaces," Applied Mathematics Letters, vol. 16, no. 7, pp. 1019-1024, 2003.

[7] A. P. Farajzadeh, M. Aslam Noor, and S. Zainab, "Mixed quasi complementarity problems in topological vector spaces," Journal of Global Optimization, vol. 45, no. 2, pp. 229-235, 2009.

[8] G. Isac, "A special variational inequality and the implicit complementarity problem," Journal of the Faculty of Science. University of Tokyo. Section IA. Mathematics, vol. 37, no. 1, pp. 109-127, 1990.

[9] N.-j. Huang and Y.-p. Fang, "Strong vector F-complementary problem and least element problem of feasible set," Nonlinear Analysis: Theory, Methods \& Applications, vol. 61, no. 6, pp. 901918, 2005. 
[10] N.-J. Huang and C.-J. Gao, "Some generalized vector variational inequalities and complementarity problems for multivalued mappings," Applied Mathematics Letters, vol. 16, no. 7, pp. 10031010, 2003.

[11] N.-j. Huang and J. Li, "F-implicit complementarity problems in Banach spaces," Zeitschrift für Analysis und ihre Anwendungen, vol. 23, no. 2, pp. 293-302, 2004.

[12] N. J. Huang, X. Q. Yang, and W. K. Chan, "Vector complementarity problems with a variable ordering relation," European Journal of Operational Research, vol. 176, no. 1, pp. 15-26, 2007.

[13] K. R. Kazmi and S. A. Khan, "Existence of solutions to a generalized system," Journal of Optimization Theory and Applications, vol. 142, no. 2, pp. 355-361, 2009.

[14] B. S. Lee, M. F. Khan, and Salahuddin, "Vector F-implicit complementarity problems with corresponding variational inequality problems," Applied Mathematics Letters, vol. 20, no. 4, pp. 433-438, 2007.

[15] J. Li and N.-j. Huang, "Vector F-implicit complementarity problems in Banach spaces," Applied Mathematics Letters, vol. 19, no. 5, pp. 464-471, 2006.

[16] U. Mosco, "Implicit variational problems and quasi variational inequalities," in Nonlinear Operators and the Calculus of Variations, vol. 543 of Lecture Notes in Mathematics, pp. 83-156, Springer, Berlin, Germany, 1976.

[17] M. A. Noor, "Mixed quasi variational inequalities," Applied Mathematics and Computation, vol. 146, no. 2-3, pp. 553-578, 2003.

[18] F. Usman and S. A. Khan, "A generalized mixed vector variational-like inequality problem," Nonlinear Analysis: Theory, Methods \& Applications, vol. 71, no. 11, pp. 5354-5362, 2009.

[19] R. U. Verma, "On generalized variational inequalities involving relaxed Lipschitz and relaxed monotone operators," Journal of Mathematical Analysis and Applications, vol. 213, no. 1, pp. 387392, 1997.

[20] X. Q. Yang, "Vector complementarity and minimal element problems," Journal of Optimization Theory and Applications, vol. 77, no. 3, pp. 483-495, 1993.

[21] C. E. Lemke, "Bimatrix equilibrium points and mathematical programming," Management Science, vol. 11, pp. 681-689, 1965.

[22] S. A. Khan, "Generalized vector implicit quasi complementarity problems," Journal of Global Optimization, vol. 49, no. 4, pp. 695-705, 2011.

[23] K. R. Kazmi, A. Khaliq, and A. Raouf, "Iterative approximation of solution of generalized mixed set-valued variational inequality problem," Mathematical Inequalities \& Applications, vol. 10, no. 3, pp. 677-691, 2007.

[24] K. R. Kazmi, F. A. Khan, and M. Shahza, “Two step algorithm for solving regularized generalized mixed variational inequality problem," Bulletin of the Korean Mathematical Society, vol. 47, no. 4, pp. 675-685, 2010.

[25] K. Fan, "A generalization of Tychonoff's fixed point theorem," Mathematische Annalen, vol. 142, pp. 305-310, 1961. 


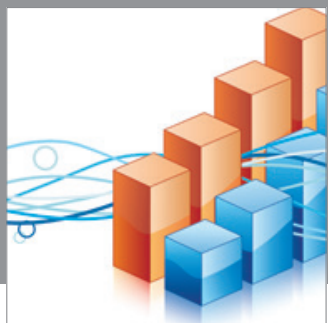

Advances in

Operations Research

mansans

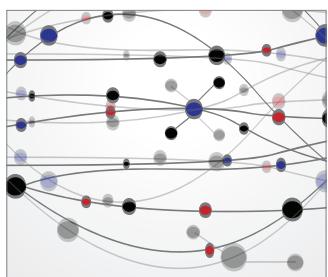

The Scientific World Journal
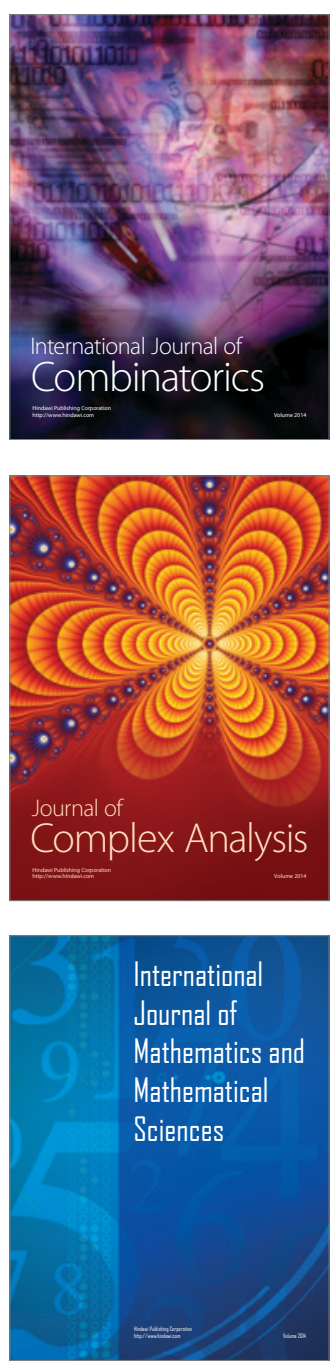
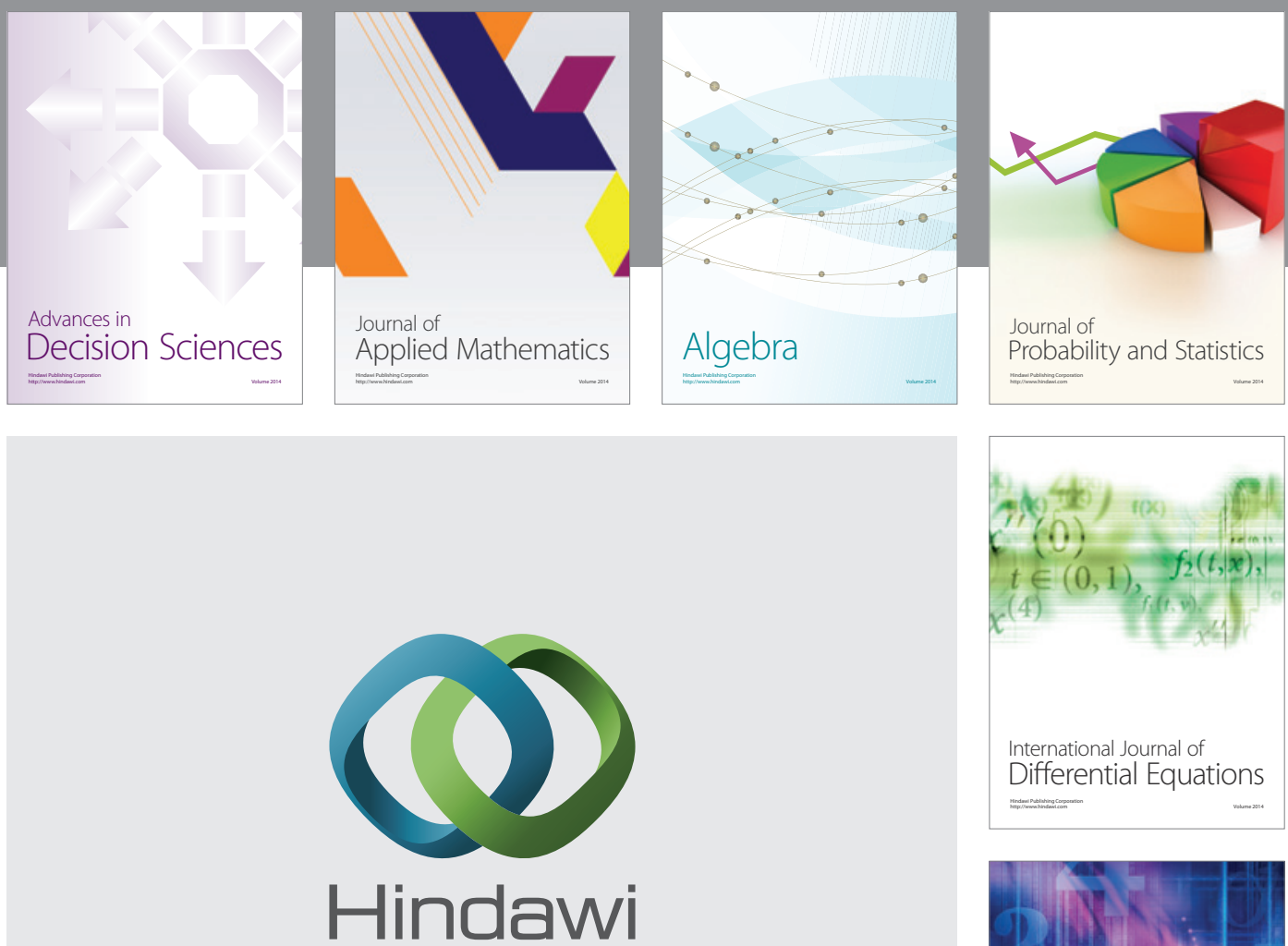

Submit your manuscripts at http://www.hindawi.com
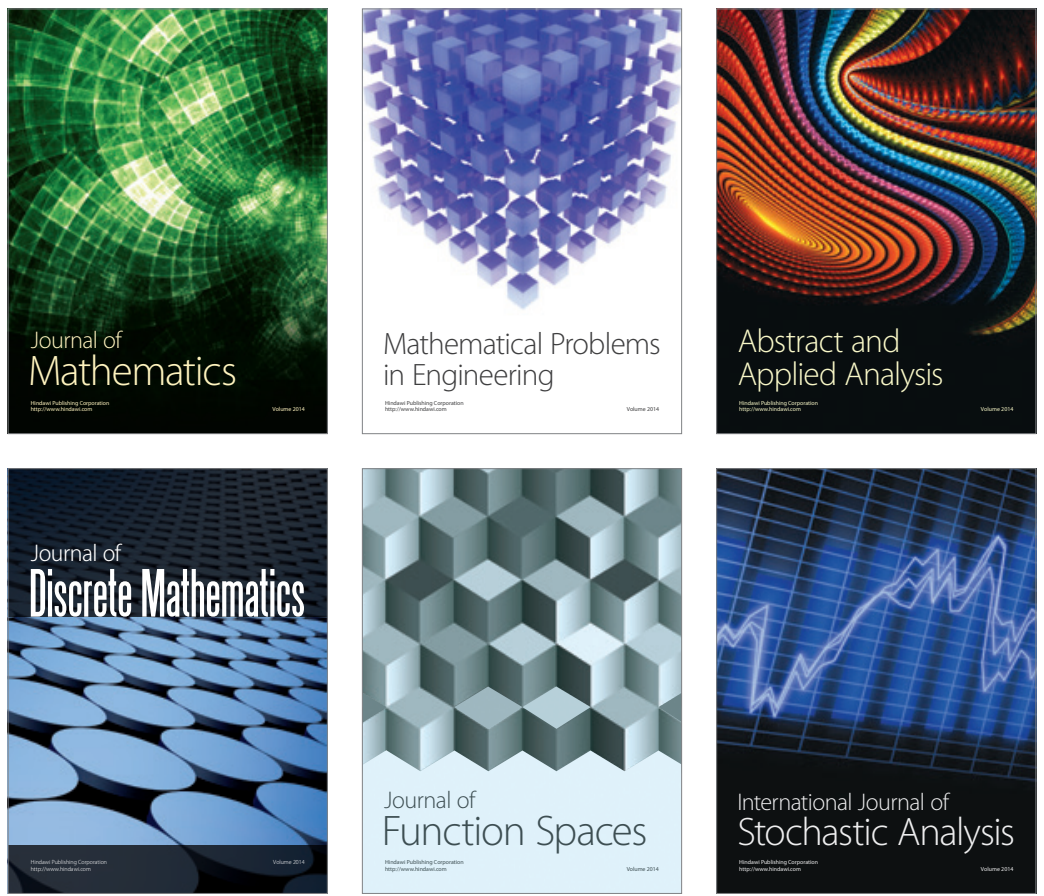

Journal of

Function Spaces

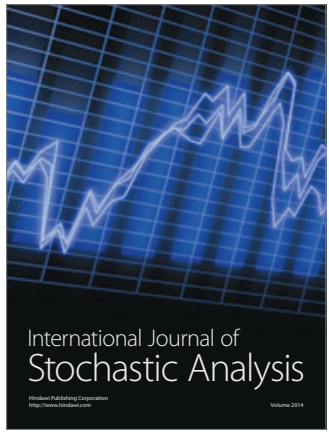

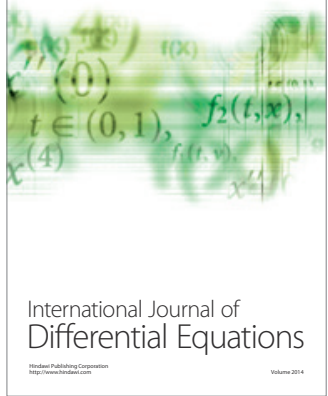
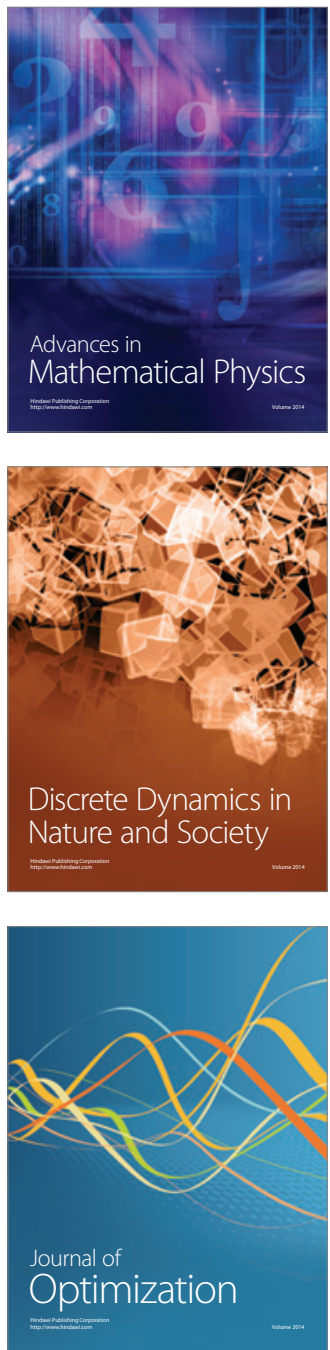